\title{
Atmospheric energy budget response to aerosol perturbation in tropical cloud systems
}

\section{Guy Dagan1, Philip Stier1, Matthew Christensen1, Guido Cioni2,3, Daniel Klocke3,4 and Axel Seifert4}

1 Atmospheric, Oceanic and Planetary Physics, Department of Physics, University of Oxford, UK

2 Max Planck Institute for Meteorology, Hamburg, Germany

3 Hans Ertel Center for Weather Research, Offenbach am Main, Germany

4 Deutscher Wetterdienst, Offenbach am Main, Germany

E-mail: guy.dagan@physics.ox.ac.uk

\section{Content:}

Figs. S1-S3.

Supplementary material containing a few examples of snapshots of MODIS-Terra (Platnick et al., 2003) true color images and cloud cover form the model output for the two cases simulated here (Figs S1-2). In addition, Fig. S3 presents relative fraction of the different cloud type for the two simulated cases.
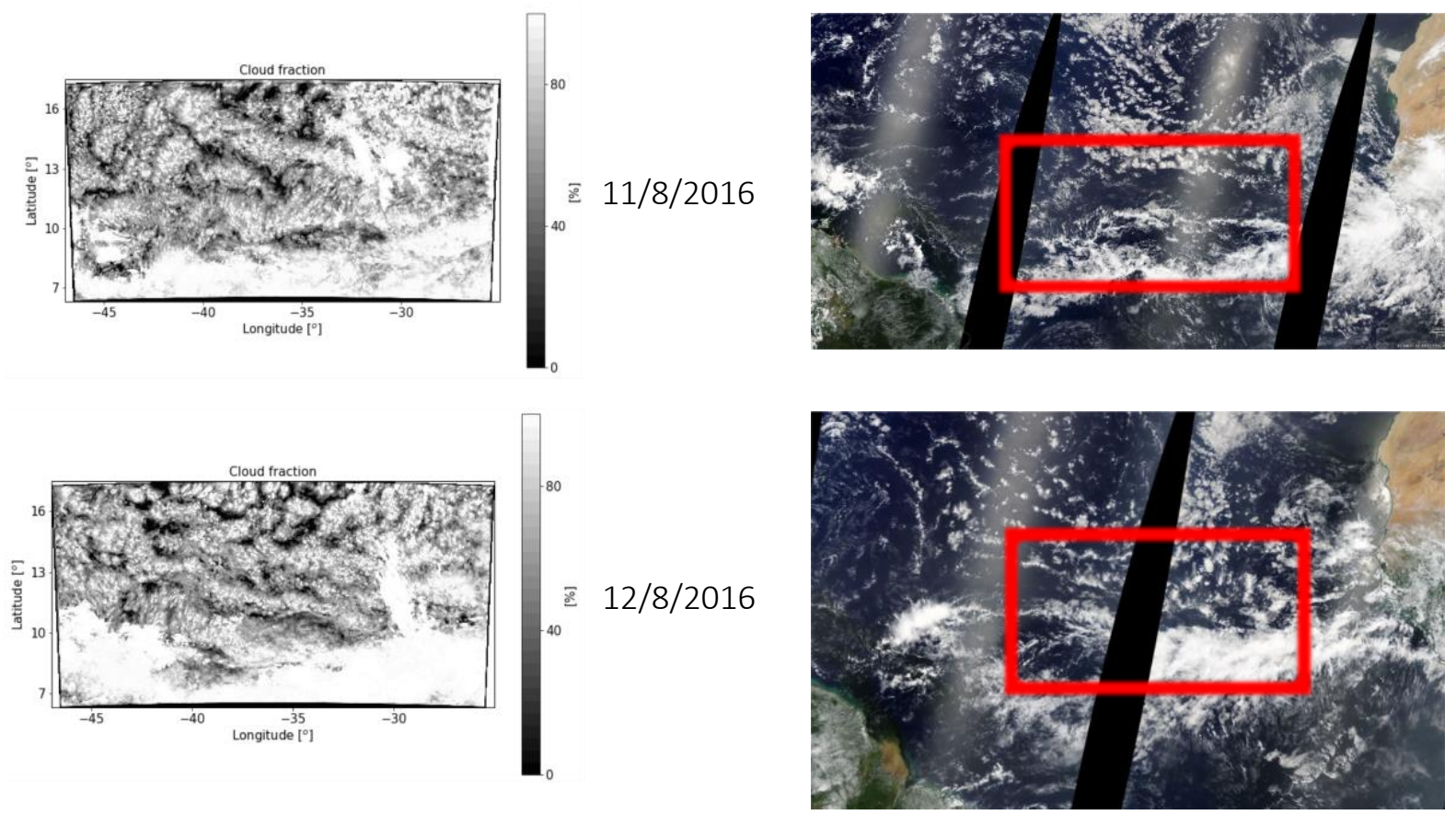
Figure S1. Cloud cover from the model simulations (left column) and MODIS-Terra true color images (right column (Platnick et al., 2003) (C) NASA) for the 11/8/2016 (upper row) and 12/8/2016 (lower row). The red rectangle represents the model domain.
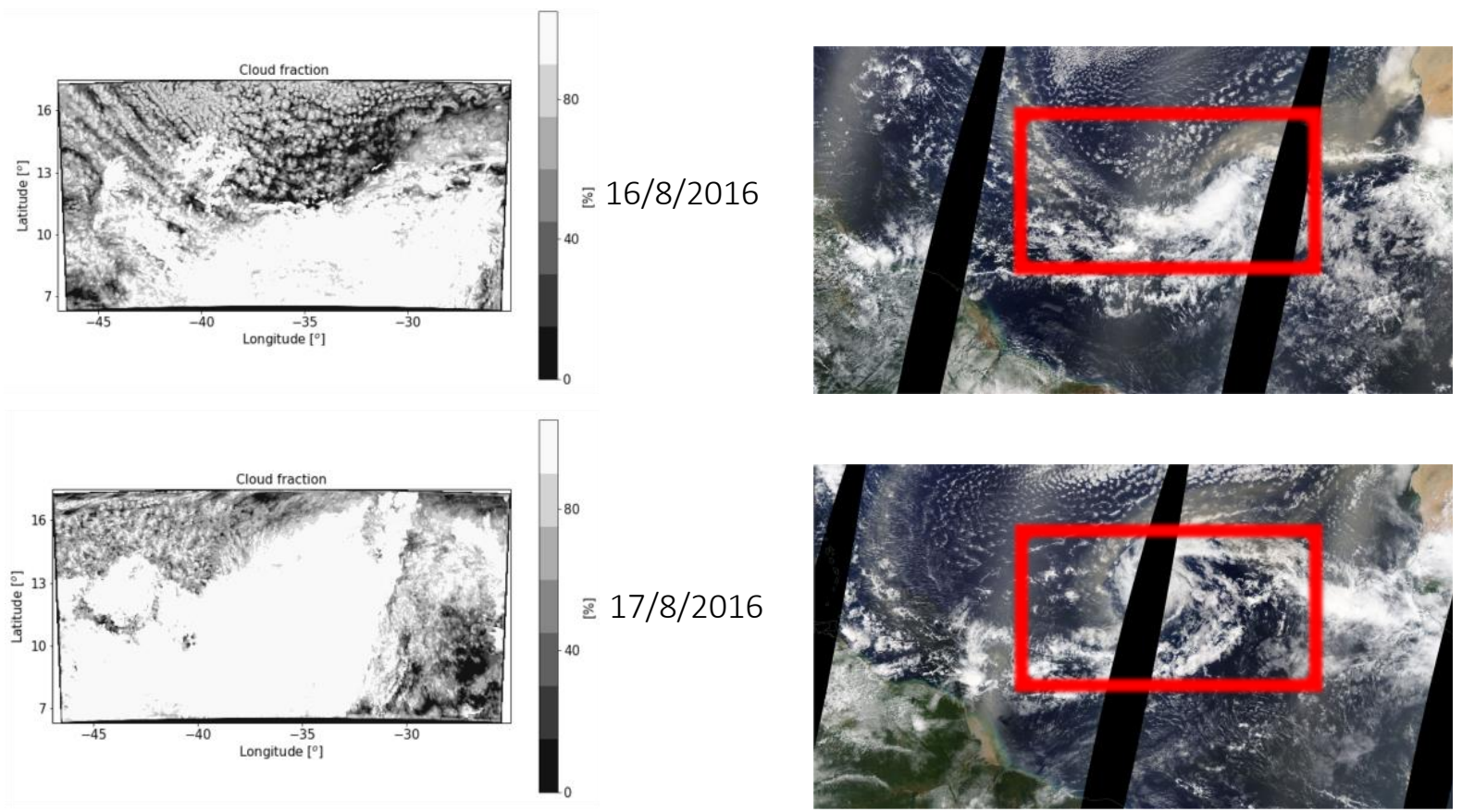

Figure S2. Cloud cover from the model simulations (left column) and MODIS-Terra true color images (right column (C) NASA) for the 16/8/2016 (upper row) and 17/8/2016 (lower row). The red rectangle represents the model domain. 


\section{Cloud classification}

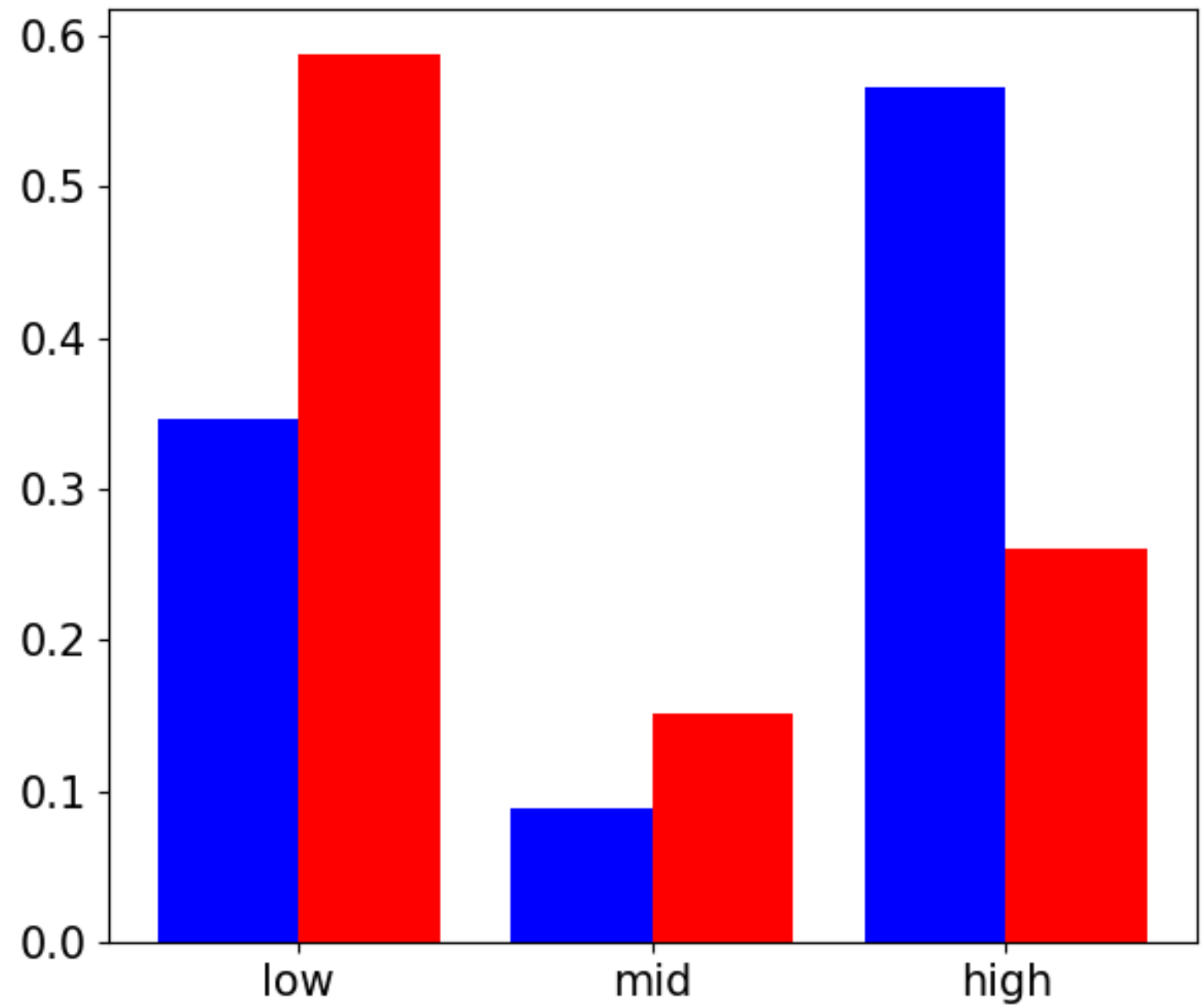

Figure S3. The relative fraction of the different cloud type for the two simulated cases. Red represents the shallow-cloud dominated case while blue represents the deep-cloud dominated case. The different cloud classifications are according to the cloud top: low is for tops below $800 \mathrm{mb}$, mid is for tops between $800 \mathrm{mb}$ and $500 \mathrm{mb}$, while high is for tops above $500 \mathrm{mb}$. In our simulations the vast majority of clouds with high top are formed by deep convection, hence the "high" category represents mostly deep convective cores and anvils. 